\title{
Some Results on Stochastic Orderings of Generalized Order Statistics and Spacings
}

\author{
Z. Zamani \\ Department of Statistics, Faculty of Mathematical Sciences, Ferdowsi University of Mashhad \\ Mashhad, Iran \\ Z.Zamani.62@gmail.com \\ G. R. Mohtashami Borzadaran \\ Department of Statistics, Faculty of Mathematical Sciences, Ferdowsi University of Mashhad \\ Mashhad, Iran \\ grmohtashami@um.ac.ir \\ M. Amini \\ Department of Statistics, Faculty of Mathematical Sciences, Ferdowsi University of Mashhad \\ Mashhad, Iran \\ m-amini@um.ac.ir
}

Received 28 November 2015

Accepted 27 November 2016

\begin{abstract}
Generalized order statistics unify the study of order statistics, record values, $k$-records, Pfeifer's records and several other cases of ordered random variables. In this paper, we first provide several comparison results for generalized order statistics in terms of the dynamic cumulative residual quantile entropy order. We also prove that when the minimum of random vectors of generalized order statistics is increasing mean residual life (IMRL), the spacings of generalized order statistics are ordered by variance, and the covariance between successive spacings is nonnegative. The normalized spacings of generalized order statistics from two sample sequences are also compared with respect to the mean residual life and the excess wealth orders.
\end{abstract}

Keywords: Generalized order statistics; Mean residual life order; Excess wealth order; Dynamic cumulative residual quantile entropy order; DFR; IFR; DMRL; IMRL.

2010 Mathematics Subject Classification: 60E15, 60K10, 62G30

\section{Introduction}

Order statistics and record values have found important applications in several fields of science and engineering; see for example, [5] and [6]. Since there exist some intimate relations among distributional, structural and dependence properties of some types of ordered random variables, it is interesting to introduce a general model containing them as special cases. To this end, the concept of generalized order statistics is introduced in [21] and [22] which includes random vectors of order statistics, record values, $k$-records, Pfeifer's records, sequential order statistics and 
progressively censored order statistics, as particular cases. Ordinary order statistics and the corresponding sample spacings play important roles in many areas of statistics such as goodness-of-fit test, auction theory, life testing and reliability. Interested readers may refer to [5], [6], [33] and [31] for discussions on this issue. Stochastic comparisons between ordinary order statistics and sample spacings are interesting topics investigated in the literature by various authors. We refer the readers to [28] and [7] for reviews of the recent developments on the topic of stochastic comparisons of order statistics and sample spacings. Because of generality of generalized order statistics, several results on stochastic comparisons of ordinary order statistics and the corresponding sample spacings have been extended to generalized order statistics and their spacings. Interested readers may refer to [17], [9], [20], [25], [16], [34], [39], [40] and [10] for a brief review. In this paper, we follow this idea and try to generalize some ordering results in the literature from ordinary order statistics and their sample spacings to generalized order statistics and the corresponding spacings. A new stochastic order, called dynamic cumulative residual quantile entropy order, is introduced in [23]. some characterizations, closure and reversed closure properties of this new order is also obtained in [23]. For ordinary order statistics, in [26] and [27] is shown that if the first order statistics has an increasing mean residual life function, then (i) the corresponding sample spacings also have increasing mean residual life functions; (ii) they can be ordered with respect to mean residual life and variance orders; and (iii) the covariance between the successive spacings is nonnegative. The generalized spacings of ordinary order statistics are compared in one-sample and two-sample problems in [19]. In particular, the mean residual life order between the normalized spacings of $X$ - and $Y$-samples is proved in [19]. The purpose of this paper is to generalize some results on order statistics obtained by [26], [27], [19] and [23] to the case of generalized order statistics. The organization of this paper is as follows: In Section 2, we briefly review some concepts that will be used later. In Section 3, we present several results concerning the comparison of generalized order statistics in terms of the dynamic cumulative residual quantile entropy order and provide a general result when the dynamic cumulative residual quantile entropy order of the minimums of random vectors of generalized order statistics implies the dynamic cumulative residual quantile entropy order of populations. We also prove that the dispersive and the convex transform orders of populations implies the dynamic cumulative residual quantile entropy order between $r$ th generalized order statistics from corresponding populations. Finally, in Section 4, we prove that under suitable conditions, the simple spacings of generalized order statistics are ordered by variance and that the covariance between successive simple spacings of generalized order statistics is nonnegative. We also study the preservation of the mean residual life order between the normalized spacings of generalized order statistics based on two different distributions and give examples to illustrate situations in which the theorems in this section are applicable.

Throughout this paper, the terms "increasing" and "decreasing" mean "non-decreasing" and "nonincreasing", respectively. All integrals and expectations are implicitly assumed to exist whenever they are written. Also, all distribution functions under consideration are restricted to be absolutely continuous with support in the positive real line $\mathbb{R}^{+}$.

\section{Preliminaries}

In this section, we first briefly review the definition of generalized order statistics, given by [21], [22] and then recall some stochastic orders and ageing notions. Finally, we state one proposition and two lemmas given in [37], [40] and [8], respectively, which will be needed later. 


\subsection{Generalized order statistics}

Generalized order statistics have been of interest in the past twenty years because they are more flexible in reliability theory, statistical modeling and inference; see, e.g., [32], [24], [18] and [15]. Uniform generalized order statistics are defined via some joint density function on a cone of the $\mathbb{R}^{n}$. Generalized order statistics based on an arbitrary distribution function $F$ are defined by means of the inverse function of $F$.

Definition 2.1. ( [21]). Let $n \in \mathbb{N}, k \geq 1, m_{1}, \cdots, m_{n-1} \in \mathbb{R}$, be parameters such that $\gamma_{n, n}=k$, $\gamma_{r, n}=k+\sum_{j=r}^{n-1}\left(m_{j}+1\right) \geq 1$, for $r=1, \cdots, n-1$ and let $\widetilde{m}_{n}=\left(m_{1}, \cdots, m_{n-1}\right)$ if $n \geq 2\left(\widetilde{m}_{n}\right.$ arbitrary if $n=1)$. If the random variables $U_{\left(r, n, \widetilde{m}_{n}, k\right)}, r=1, \cdots, n$ possess a joint density of the form

$$
f_{U_{\left(1, n, \tilde{m}_{n}, k\right)}, \cdots, U_{\left(n, n, \tilde{m}_{n}, k\right)}}\left(u_{1}, \cdots, u_{n}\right)=k\left(\prod_{j=1}^{n-1} \gamma_{j, n}\right)\left(\prod_{i=1}^{n-1}\left(1-u_{i}\right)^{m_{i}}\right)\left(1-u_{n}\right)^{k-1},
$$

on the cone $0 \leq u_{1} \leq u_{2} \leq \cdots \leq u_{n}<1$ of $\mathbb{R}^{n}$, they are called uniform generalized order statistics. If $F$ is an arbitrary distribution function, then the random variables

$$
X_{\left(r, n, \widetilde{m}_{n}, k\right)}=F^{-1}\left(U_{\left(r, n, \widetilde{m}_{n}, k\right)}\right), \quad r=1, \cdots, n,
$$

are called the generalized order statistics based on $F$, where $F^{-1}$ is the inverse of $F$ defined by $F^{-1}(u)=\sup \{x: F(x) \leq u\}$ for $u \in[0,1]$.

In the particular case $m_{1}=\cdots=m_{n-1}=m$, we write $U_{(r, n, m, k)}$ and $X_{(r, n, m, k)}, r=1, \cdots, n$, respectively.

If $F$ is absolutely continuous with density function $f$, the marginal density and distribution functions of $r$ th generalized order statistics, $X_{\left(r, n, \widetilde{m}_{n}, k\right)}$, for $r=1, \cdots, n$ are given by

$$
f_{X_{\left(r, n, \tilde{m}_{n}, k\right)}}(x)=c_{r-1, n} f(x) \sum_{i=1}^{r} a_{i}(1-F(x))^{\gamma_{i, n}-1},
$$

and

$$
F_{X_{\left(r, n, \tilde{m}_{n}, k\right)}}(x)=1-c_{r-1, n} \sum_{i=1}^{r} \frac{a_{i}}{\gamma_{i, n}}(1-F(x))^{\gamma_{i, n}}
$$

where $a_{i}=\prod_{j=1, j \neq i}^{r} \frac{1}{\gamma_{j, n}-\gamma_{i, n}}$ and $c_{r-1, n}=\prod_{j=1}^{r} \gamma_{j, n}$. These functions for $X_{(r, n, m, k)}$ have closed forms as follows:

$$
f_{X_{(r, n, m, k)}}(x)=\phi_{r: n}(F(x)) f(x),
$$

and

$$
F_{X_{(r, n, m, k)}}(x)=\psi_{r: n}(F(x)),
$$

where

$$
\phi_{r: n}(u)=\frac{c_{r-1, n}}{(r-1) !}(1-u)^{\gamma_{r, n}-1}\left[\delta_{m}(u)\right]^{r-1}, u \in[0,1]
$$




$$
\psi_{r: n}(u)=1-\sum_{i=0}^{r-1} \frac{c_{i-1, n}}{i !}(1-u)^{\gamma_{i+1, n}}\left[\delta_{m}(u)\right]^{i}, u \in[0,1] .
$$

Here, the function $\delta_{m}:[0,1) \longrightarrow \mathbb{R}, m \in \mathbb{R}$ is defined by

$$
\delta_{m}(x)= \begin{cases}\frac{1}{m+1}\left[1-(1-x)^{m+1}\right], & m \neq-1, \\ -\ln (1-x), & m=-1 .\end{cases}
$$

Generalized order statistics provide an unified approach for examining distributional and moment properties of many models of ordered random variables, which can actually be regarded as special cases. For example, ordinary order statistics of a random sample from a distribution $F$ are a particular case of generalized order statistics when $k=1$ and $m_{r}=0$ for all $r=1, \cdots, n-1$. When $k=1$ and $m_{1}=\cdots=m_{n-1}=-1$, then we get the first $n$ record values from a sequence of random variables with distribution $F$. Choosing the parameters appropriately, the model of generalized order statistics also yields order statistics with non-integral sample size, $k$-record values, sequential order statistics, Pfeifer's records, progressive Type II censored order statistics, order statistics under multivariate imperfect repair, etc., as special cases. For more details, one may refer to [22], [4] and [9]. It is well-known that generalized order statistics from a continuous distribution form a Markov chain with transition probabilities

$$
P\left[X_{\left(r, n, \widetilde{m}_{n}, k\right)}>t \mid X_{\left(r-1, n, \widetilde{m}_{n}, k\right)}=s\right]=\left[\frac{\bar{F}(t)}{\bar{F}(s)}\right]^{\gamma_{r, n}},
$$

for $t \geq s$ and $r=2, \cdots, n$.

Let $X$ and $Y$ be two nonnegative random variables with associated distribution functions $F$ and $G$, and let $\left\{X_{\left(i, n, \widetilde{m}_{n}, k\right)}, i=1, \cdots, n\right\}$ and $\left\{Y_{\left(j, m, \widetilde{m}_{m}, k\right)}, j=1, \cdots, m\right\}$, be generalized order statistics based on $F$ and $G$, respectively. The random variables $U_{i, n}=X_{\left(i, n, \widetilde{m}_{n}, k\right)}-X_{\left(i-1, n, \widetilde{m}_{n}, k\right)}$ and $\widetilde{U}_{i, n}=\gamma_{i, n} U_{i, n}, i=$ $1, \cdots, n$, with $X_{\left(0, n, \widetilde{m}_{n}, k\right)} \equiv 0$, are the simple spacings and the normalized spacings of the generalized order statistics $\left\{X_{\left(i, n, \widetilde{m}_{n}, k\right)}, i=1, \cdots, n\right\}$. Similarly, define $V_{j, m}=Y_{\left(j, m, \widetilde{m}_{m}, k\right)}-Y_{\left(j-1, m, \widetilde{m}_{m}, k\right)}$ and $\widetilde{V}_{j, m}=$ $\gamma_{j, m} V_{j, m}$ for $j=1, \cdots, m$, where $Y_{\left(0, m, \widetilde{m}_{m}, k\right)} \equiv 0$.

The conditional survival function of $[X-y \mid X>y]$, where $X$ is a nonnegative random variable with distribution function $F$ is denoted by $\bar{F}_{y}(x)=\frac{\bar{F}(y+x)}{\bar{F}(y)}$ for $x \in \mathbb{R}^{+}$.

\subsection{Some ageing notions and stochastic orders}

The exact distribution of the life of an unit or a system is often unavailable in practice, but nonparametric ageing classes of life distributions, such as increasing (decreasing) failure rate and increasing (decreasing) mean residual life have been found to be quite useful in maintain policy and system analysis.

Some stochastic orders and classes of the life distributions have defined in [37] as follows. Let $X$ and $Y$ be two nonnegative random variables with absolutely continuous distribution functions $F$ and $G$ and densities $f$ and $g$, respectively. Also, denote their respective survival functions by $\bar{F}=1-F$ and $\bar{G}=1-G$ and their mean residual life functions by $\mu_{X}(t)$ and $\mu_{Y}(t)$, respectively.

Definition 2.2. A random variable $X$ is said to have an increasing (decreasing)

(i) failure rate (IFR (DFR)), if the hazard rate function of $X$, i.e. $r_{X}(t)=\frac{f(t)}{F(t)}$ is increasing (decreasing) in $t>0$. 
(ii) mean residual life (IMRL (DMRL)), if the mean residual life of $X$, i.e.

$$
\mu_{X}(t)=E(X-t \mid X>t)=\frac{\int_{t}^{\infty} \bar{F}(u) d u}{\bar{F}(t)},
$$

is increasing (decreasing) in $t$.

It is well known (see [8]) that

$$
\operatorname{IFR}(\mathrm{DFR}) \Longrightarrow D M R L(I M R L) .
$$

Definition 2.3. A random variable $X$ is smaller than a random variable $Y$ in the

(i) mean residual life order (denoted by $X \leq_{m r l} Y$ ), if $E X$ and $E Y$ exist and $\mu_{X}(t) \leq \mu_{Y}(t)$ for all $t$.

(ii) harmonic mean residual life order (denoted by $X \leq_{h m r l} Y$ ), if

$$
\left[\frac{1}{t} \int_{0}^{t} \frac{1}{\mu_{Y}(x)} d x\right]^{-1} \leq\left[\frac{1}{t} \int_{0}^{t} \frac{1}{\mu_{X}(x)} d x\right]^{-1}
$$

for all $t>0$.

(iii) excess wealth order (denoted by $X \leq_{e w} Y$ ), if

$$
\int_{F^{-1}(p)}^{\infty} \bar{F}(x) d x \leq \int_{G^{-1}(p)}^{\infty} \bar{G}(x) d x,
$$

where $F^{-1}$ is the inverse of $F$ defined by $F^{-1}(u)=\sup \{x: F(x) \leq u\}$ for $u \in[0,1)$ and $p$ belong to $(0,1)$.

(iv) dispersive order (denoted by $X \leq_{\text {disp }} Y$ ), if $F^{-1}(\beta)-F^{-1}(\alpha) \leq G^{-1}(\beta)-G^{-1}(\alpha)$ whenever $0<\alpha \leq \beta<1$, where $F^{-1}$ and $G^{-1}$ are the right-continuous inverse of $F$ and $G$, respectively.

(v) convex transform order (denoted by $X \leq_{c} Y$ ) if $G^{-1}[F(x)]$ is convex, or equivalently, $f(x) / g\left[G^{-1}(F(x))\right]$ is increasing in $x>0$.

(vi) increasing convex order (denoted by $X \leq_{i c x} Y$ ) if and only if

$$
\int_{t}^{\infty} \bar{F}(x) d x \leq \int_{t}^{\infty} \bar{G}(x) d x, \text { for all } t .
$$

For a nonnegative random variable $X$ with survival function $\bar{F}$, a new dynamic measure of uncertainty, called the dynamic cumulative residual quantile entropy (DCRQE), is proposed in [23] which is defined as follows:

$$
\phi_{X}(p)=-\int_{F^{-1}(p)}^{\infty} \frac{\bar{F}(x)}{1-p} \ln \frac{\bar{F}(x)}{1-p} d x, \text { for all } p \in(0,1) .
$$

Also a new stochastic order based on DCRQE function was introduced in [23]. The definition of this order is given in the following.

Definition 2.4. Let $X$ and $Y$ be two nonnegative absolutely continuous random variables with DCRQE functions $\phi_{X}(p)$ and $\phi_{Y}(p)$, respectively. $X$ is said to be smaller than $Y$ in the dynamic cumulative residual quantile entropy order (denoted by $X \leq_{D C R Q E} Y$ ), if $\phi_{X}(p) \leq \phi_{Y}(p)$ for all $p \in(0,1)$. 
It was proved that $X \leq_{D C R Q E} Y$ if, and only if,

$$
\int_{t}^{\infty} \bar{F}(x) \ln \left[\frac{\bar{F}(t)}{\bar{F}(x)}\right]\left[\frac{f(x)}{g\left[G^{-1}(F(x))\right]}-1\right] d x \geq 0, \text { for all } t \geq 0 .
$$

For more details see [23].

The relationship between the above orderings are shown in the following diagram:

$$
\begin{aligned}
X \leq_{c} Y, \frac{f(0)}{g(0)} \geq 1 \Longrightarrow X \leq_{D C R Q E} Y \Longleftarrow X \leq_{\text {disp }} Y \\
\Uparrow_{X \leq_{e w} Y} \Longrightarrow X \leq_{i c x} Y
\end{aligned}
$$

and

$$
X \leq_{m r l} Y \Longrightarrow X \leq_{h m r l} Y .
$$

See [37] and [23] for more details on their interrelationships and properties. The next proposition is due to [37] (Theorem 3.C.6), and shows that the mean residual life order can be characterized by the excess wealth order.

Proposition 2.1. Let $X$ and $Y$ be two random variables with distribution functions $F$ and $G$, respectively. If $X \leq_{m r l} Y$ and if either $X$ or $Y$ or both are IMRL, then $X \leq_{e w} Y$.

The following lemma will be used in deriving the main results in Section 4.

Lemma 2.1. ([40]). Let $X_{\left(1, n, \widetilde{m}_{n}, k\right)}, \cdots, X_{\left(n, n, \widetilde{m}_{n}, k\right)}$ be generalized order statistics based on $F$, where $m_{i} \geq-1$ for each $i$, and denote $\widetilde{m}_{r, n}^{*}=\left(m_{r+1}, \cdots, m_{n-1}\right)$ for $r=1, \cdots, n-2$.

(i) If $X_{\left(1, n, \widetilde{m}_{n}, k\right)}$ is IMRL (DMRL), then $X_{\left(1, n-r, \widetilde{m}_{r, n}^{*}, k\right)}$ is IMRL (DMRL) for each $r$.

(ii) If $X_{\left(1, n, \widetilde{m}_{n}, k\right)}$ is IMRL, then $U_{r, n}$ is IMRL for each $r$.

Some of the results of the paper are based on the following technical lemma which is essentially due to [8].

Lemma 2.2. Let $W(x)$ be a Lebesgue-Stieltjes measure, not necessarily positive and let $h(x)$ be a nonnegative real function. If $h$ is increasing and $\int_{t}^{\infty} d W(x) \geq 0$, for all $t \in \mathbb{R}$, then $\int_{-\infty}^{\infty} h(x) d W(x) \geq$ 0 .

\section{Comparison of generalized order statistics in the dynamic cumulative residual quantile entropy order}

In this section, we obtain some results concerning the dynamic cumulative residual quantile entropy order of generalized order statistics. The following theorem provides a general result when the dynamic cumulative residual quantile entropy order of the minimums of random vectors of generalized order statistics implies the dynamic cumulative residual quantile entropy order of the populations. This theorem generalizes Theorem 3.2 in [23] from ordinary order statistics to generalized order statistics.

Theorem 3.1. Let $\left\{X_{\left(r, n, \widetilde{m}_{n}, k\right)}, r=1, \cdots, n\right\}$ and $\left\{Y_{\left(r, n, \widetilde{m}_{n}, k\right)}, r=1, \cdots, n\right\}$ be generalized order statistics based on absolutely continuous distribution functions $F$ and $G$, respectively. If $X_{\left(1, n, \widetilde{m}_{n}, k\right)} \leq_{D C R Q E} Y_{\left(1, n, \widetilde{m}_{n}, k\right)}$, then $X \leq_{D C R Q E} Y$. 
Proof. Suppose assumption $X_{\left(1, n, \widetilde{m}_{n}, k\right)} \leq_{D C R Q E} Y_{\left(1, n, \widetilde{m}_{n}, k\right)}$ holds. From (2.2), we have

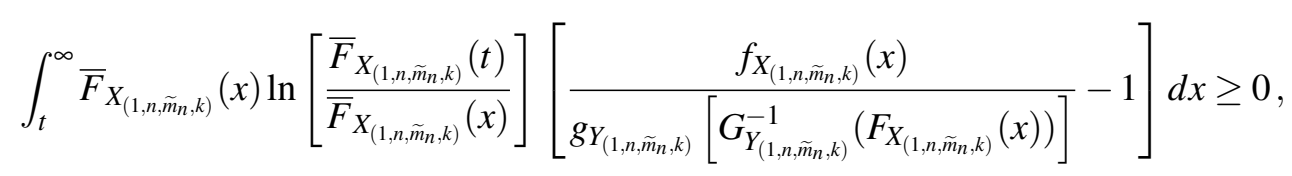

for all $t \geq 0$. We know that $\bar{F}_{X_{\left(1, n, \tilde{m}_{n}, k\right)}}(x)=(\bar{F}(x))^{\gamma_{1, n}}, f_{X_{\left(1, n, \tilde{m}_{n}, k\right)}}(x)=\gamma_{1, n}(\bar{F}(x))^{\gamma_{1, n}-1} f(x)$, and similarly $g_{Y_{\left(1, n, \tilde{m}_{n}, k\right)}}(x)=\gamma_{1, n}(\bar{G}(x))^{\gamma_{1, n}-1} g(x)$. It is obvious that

$$
G_{Y_{\left(1, n, \tilde{m}_{n}, k\right)}^{-1}}^{-}(x)=G^{-1}\left(1-(1-x)^{\frac{1}{\gamma_{1, n}}}\right)
$$

so,

$$
G_{Y_{\left(1, n, \tilde{m}_{n}, k\right)}^{-1}}^{-1}\left(F_{X_{\left(1, n, \tilde{m}_{n}, k\right)}}(x)\right)=G^{-1}(F(x)) .
$$

Therefore,

$$
\frac{f_{X_{\left(1, n, \tilde{m}_{n}, k\right)}}(x)}{g_{Y_{\left(1, n, \tilde{m}_{n}, k\right)}}\left[G_{Y_{\left(1, n, \tilde{m}_{n}, k\right)}}^{-1}\left(F_{X_{\left(1, n, \tilde{m}_{n}, k\right)}}(x)\right)\right]}=\frac{f(x)}{g\left[G^{-1}(F(x))\right]} .
$$

But

$$
\ln \left[\frac{\bar{F}_{X_{\left(1, n, \tilde{m}_{n}, k\right)}(t)}}{\bar{F}_{X_{\left(1, n, \tilde{m}_{n}, k\right)}}(x)}\right]=\gamma_{1, n} \ln \left[\frac{\bar{F}(t)}{\bar{F}(x)}\right]
$$

Substituting (3.2) and (3.3) in (3.1), we have

$$
\int_{t}^{\infty} \gamma_{1, n}[\bar{F}(x)]^{\gamma_{1, n}-1} \bar{F}(x) \ln \left[\frac{\bar{F}(t)}{\bar{F}(x)}\right]\left[\frac{f(x)}{g\left[G^{-1}(F(x))\right]}-1\right] d x \geq 0
$$

The function

$$
h(x)=\left[\gamma_{1, n}(\bar{F}(x))^{\gamma_{1, n}-1}\right]^{-1},
$$

is nonnegative and increasing in $x \geq 0$, because $\gamma_{1, n} \geq 1$ and $\bar{F}(x)$ is decreasing in $x \geq 0$. By (3.4), (3.5) and Lemma 2.2, we see that

$$
\int_{t}^{\infty} \bar{F}(x) \ln \left[\frac{\bar{F}(t)}{\bar{F}(x)}\right]\left[\frac{f(x)}{g\left[G^{-1}(F(x))\right]}-1\right] d x \geq 0, \text { for all } t \geq 0
$$

holds, which proves the theorem. 
Remark 3.1. Counterexample 3.1 in [23] shows that

$$
X \leq_{D C R Q E} Y \Rightarrow X_{1: n} \leq_{D C R Q E} Y_{1: n}, X_{1: n} \geq_{D C R Q E} Y_{1: n} .
$$

In the following, we consider this Counterexample to show that

$$
X \leq_{D C R Q E} Y \Longrightarrow X_{r: n} \leq_{D C R Q E} Y_{r: n}, \text { for } r=2, \cdots, n-1 .
$$

Example 3.1. Let $X$ and $Y$ be two nonnegative continuous random variables with distribution functions

$$
F(x)=x, x \in(0,1), \text { and } G(x)= \begin{cases}2 x, & x \in\left[0, \frac{1}{4}\right) \\ \frac{2}{3} x+\frac{1}{3}, & x \in\left[\frac{1}{4}, 1\right)\end{cases}
$$

respectively. We have $F_{X_{r: n}}(x)=D_{r: n}(F(x))$ and $f_{X_{r: n}}(x)=f(x) E_{r: n}(F(x))$, where

$$
D_{r: n}(u)=\sum_{k=r}^{n}\left(\begin{array}{l}
n \\
k
\end{array}\right) u^{k}(1-u)^{n-k}, \text { and } E_{r: n}(u)=n\left(\begin{array}{c}
n-1 \\
r-1
\end{array}\right) u^{r-1}(1-u)^{n-r} .
$$

It is obvious that for all $x \geq 0$,

$$
\frac{f_{X_{r: n}}(x)}{g_{Y_{r: n}}\left[G_{Y_{r: n}}^{-1}\left(F_{X_{r: n}}(x)\right)\right]}=\frac{f(x)}{g\left[G^{-1}(F(x))\right]} .
$$

We want to show that $X_{2: 4} \not{ }_{D C R Q E} Y_{2: 4}$. Therefore

$$
\begin{aligned}
I_{s}(t) & =\int_{t}^{\infty} \bar{F}_{X_{2: 4}}(x) \ln \left[\frac{\bar{F}_{X_{2: 4}}(t)}{\bar{F}_{X_{2: 4}}(x)}\right]\left[\frac{f_{X_{2: 4}}(x)}{g_{Y_{2: 4}}\left[G_{Y_{2: 4}}^{-1}\left(F_{X_{2: 4}}(x)\right)\right]}-1\right] d x \\
& =\int_{t}^{1}(1-x)^{3}(1+3 x) \ln \left[\frac{(1-t)^{3}(1+3 t)}{(1-x)^{3}(1+3 x)}\right]\left[\frac{f(x)}{g\left[G^{-1}(F(x))\right]}-1\right] d x .
\end{aligned}
$$

If $t \in\left[0, \frac{1}{2}\right)$, then

$$
\begin{aligned}
I_{s}(t) & =-\frac{1}{2} \int_{t}^{\frac{1}{2}}(1-x)^{3}(1+3 x) \ln \left[\frac{(1-t)^{3}(1+3 t)}{(1-x)^{3}(1+3 x)}\right] d x \\
& +\frac{1}{2} \int_{\frac{1}{2}}^{1}(1-x)^{3}(1+3 x) \ln \left[\frac{(1-t)^{3}(1+3 t)}{(1-x)^{3}(1+3 x)}\right] d x \\
& =\frac{1}{4050}\left[-177.186 \ln \left((1-t)^{3}(1+3 t)\right)+2104.064\right. \\
& +972(1-t)^{5}-1620(1-t)^{4}-180(1-t)^{3} \\
& \left.-360(1-t)^{2}-960(1-t)-1280 \ln (1+3 t)\right] .
\end{aligned}
$$

If $t \in\left[\frac{1}{2}, 1\right)$, then

$$
\begin{aligned}
I_{s}(t) & =\frac{1}{2} \int_{t}^{1}(1-x)^{3}(1+3 x) \ln \left[\frac{(1-t)^{3}(1+3 t)}{(1-x)^{3}(1+3 x)}\right] d x \\
& =\frac{1}{2}\left[-1774.456-972(1-t)^{5}+1620(1-t)^{4}\right. \\
& \left.+180(1-t)^{3}+360(1-t)^{2}+960(1-t)+1280 \ln (1+3 t)\right] \\
& \geq 0 .
\end{aligned}
$$


Therefore for $t=0, I_{S}(t)=-\frac{43.936}{4050}<0$ and for $t \in\left[\frac{1}{2}, 1\right), I_{S}(t) \geq 0$, i.e.

$$
X_{2: 4} \not{ }_{D C R Q E} Y_{2: 4} \text {, and } X_{2: 4} \nsupseteq D C R Q E Y_{2: 4} \text {. }
$$

Remark 3.2. Using Remark 3.1 and Example 3.1, we conclude that:

$$
X \leq_{D C R Q E} Y \Rightarrow X_{\left(r, n, \widetilde{m}_{n}, k\right)} \leq_{D C R Q E} Y_{\left(r, n, \widetilde{m}_{n}, k\right)}, \text { for } r=1, \cdots, n-1 .
$$

The following result is concerning to the minimum from two vectors of generalized order statistics.

Theorem 3.2. Let $\left\{X_{\left(r, n, \widetilde{m}_{n}, k\right)}, r=1, \cdots, n\right\}$ and $\left\{Y_{\left(r, n, \widetilde{m}_{n}, k\right)}, r=1, \cdots, n\right\}$ be generalized order statistics based on continuous distribution functions $F$ and $G$, respectively, with parameter $\gamma_{1, n}=$ $k+\sum_{j=1}^{n-1}\left(m_{j}+1\right)$. Similarly, let $\left\{X_{\left(r, n^{\prime}, \tilde{m}_{n^{\prime}}^{\prime}, k\right)}, r=1, \cdots, n^{\prime}\right\}$ and $\left\{Y_{\left(r, n^{\prime}, \tilde{m}_{n^{\prime}}^{\prime}, k\right)}, r=1, \cdots, n^{\prime}\right\}$ be generalized order statistics based on $F$ and $G$, respectively, with parameter $\gamma_{1, n^{\prime}}^{\prime}=k+\sum_{j=1}^{n^{\prime}-1}\left(m_{j}^{\prime}+1\right)$. If $\gamma_{1, n} \leq \gamma_{1, n^{\prime}}^{\prime}$ and $X_{\left(1, n, \widetilde{m}_{n}, k\right)} \leq_{D C R Q E} Y_{\left(1, n, \widetilde{m}_{n}, k\right)}$, then $X_{\left(1, n^{\prime}, \widetilde{m}_{n^{\prime}}^{\prime}, k\right)} \leq_{D C R Q E} Y_{\left(1, n^{\prime}, \tilde{m}_{n^{\prime}}^{\prime}, k\right)}$.

Proof. From (2.2) and (3.2), the condition $X_{\left(1, n, \widetilde{m}_{n}, k\right)} \leq{ }_{D C R Q E} Y_{\left(1, n, \widetilde{m}_{n}, k\right)}$ is equivalent to

$$
\int_{t}^{\infty} \gamma_{1, n}(\bar{F}(x))^{\gamma_{1, n}} \ln \left[\frac{\bar{F}(t)}{\bar{F}(x)}\right]\left[\frac{f(x)}{g\left[G^{-1}(F(x))\right]}-1\right] d x \geq 0 .
$$

Since $h(x)=\frac{\gamma_{1, n^{\prime}}^{\prime}}{\gamma_{1, n}}(\bar{F}(x))^{\gamma_{1, n^{\prime}}^{\prime}-\gamma_{1, n}}$ is a nonnegative and increasing function, from Lemma 2.2, it follows that

$$
\int_{t}^{\infty} \gamma_{1, n^{\prime}}^{\prime}(\bar{F}(x))^{\gamma_{1, n^{\prime}}^{\prime}} \ln \left[\frac{\bar{F}(t)}{\bar{F}(x)}\right]\left[\frac{f(x)}{g\left[G^{-1}(F(x))\right]}-1\right] d x \geq 0
$$

The survival function of $X_{\left(1, n^{\prime}, \tilde{m}_{n^{\prime}}^{\prime}, k\right)}$ and $Y_{\left(1, n^{\prime}, \tilde{m}_{n^{\prime}}^{\prime}, k\right)}$ are given by $\bar{F}_{X_{\left(1, n^{\prime}, \tilde{m}^{\prime} n^{\prime}, k\right)}}(x)=(\bar{F}(x))^{\gamma_{1, n^{\prime}}^{\prime}}$ and $\bar{G}_{Y_{\left(1, n^{\prime}, \tilde{m}^{\prime}{ }^{\prime}, k\right)}}(x)=(\bar{G}(x))^{\gamma_{1, n^{\prime}}^{\prime}}$, respectively. So, we have

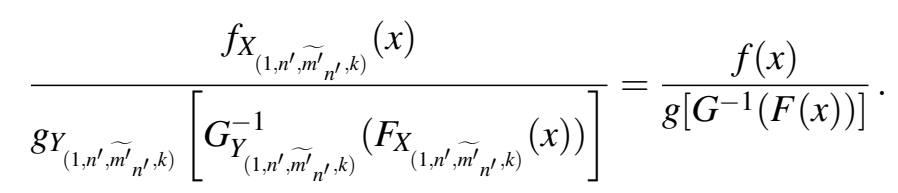

Therefore (3.6) is equivalent to $X_{\left(1, n^{\prime}, \widetilde{m}_{n^{\prime}}^{\prime}, k\right)} \leq_{D C R Q E} Y_{\left(1, n^{\prime}, \widetilde{m}_{n^{\prime}}^{\prime}, k\right)}$.

Theorem 3.3. Let $X$ and $Y$ be two nonnegative random variables with corresponding absolutely continuous distribution functions $F$ and $G$ and let $X_{\left(r, n, \widetilde{m}_{n}, k\right)}$ and $Y_{\left(r, n, \widetilde{m}_{n}, k\right)}, r=1, \cdots, n$ be generalized order statistics based on $F$ and $G$, respectively. If $X \leq_{\text {disp }} Y$, then

$$
X_{\left(r, n, \widetilde{m}_{n}, k\right)} \leq_{D C R Q E} Y_{\left(r, n, \widetilde{m}_{n}, k\right)} .
$$

Proof. Based on theorem 3.12 of [9], $X \leq_{\text {disp }} Y$ implies $X_{\left(r, n, \widetilde{m}_{n}, k\right)} \leq_{\text {disp }} Y_{\left(r, n, \widetilde{m}_{n}, k\right)}$. Furthermore, the dispersive order implies the DCRQE order (see [23]). This proves the theorem. 
Under the dispersive order between $F$ and $G$, the random vectors of generalized order statistics based on $F$ and $G$ are ordered by dispersive order (see [9]). From (2.3), we have the next corollary.

Corollary 3.1. Let $X$ and $Y$ be random variables with absolutely continuous distribution functions $F$ and $G$, respectively, and let $\mathbf{X}=\left(X_{\left(1, n, \widetilde{m}_{n}, k\right)}, \cdots, X_{\left(n, n, \widetilde{m}_{n}, k\right)}\right)$ and $\mathbf{Y}=\left(Y_{\left(1, n, \widetilde{m}_{n}, k\right)}, \cdots, Y_{\left(n, n, \widetilde{m}_{n}, k\right)}\right)$ be random vectors of generalized order statistics based on $F$ and $G$, respectively. If $X \leq_{\text {disp }} Y$, then $\mathbf{X} \leq_{D C R Q E} \mathbf{Y}$.

Theorem 3.4. Let $\left\{X_{\left(r, n, \widetilde{m}_{n}, k\right)}, r=1, \cdots, n\right\}$ and $\left\{Y_{\left(r, n, \widetilde{m}_{n}, k\right)}, r=1, \cdots, n\right\}$ be the generalized order statistics based on absolutely continuous distribution functions $F$ and $G$, respectively. If $X_{\left(1, n, \widetilde{m}_{n}, k\right)} \leq_{e w} Y_{\left(1, n, \widetilde{m}_{n}, k\right)}$, then $X \leq_{D C R Q E} Y$.

Proof. Suppose that $X_{\left(1, n, \widetilde{m}_{n}, k\right)} \leq_{e w} Y_{\left(1, n, \widetilde{m}_{n}, k\right)}$. From relation (2.3), we have

$$
X_{\left(1, n, \widetilde{m}_{n}, k\right)} \leq_{D C R Q E} Y_{\left(1, n, \widetilde{m}_{n}, k\right)} .
$$

Therefore by Theorem 3.1, we conclude that $X \leq_{D C R Q E} Y$. So, the desired result is obtained.

In the following theorem we consider the special case of generalized order statistics when $m_{i}, i=1, \cdots, n$ are equal.

Theorem 3.5. Let $\left\{X_{(r, n, m, k)}, r=1, \cdots, n\right\}$ and $\left\{Y_{(r, n, m, k)}, r=1, \cdots, n\right\}$ be m-generalized order statistics based on absolutely continuous distribution functions $F$ and $G$, respectively. If $X \leq_{c} Y$ and $\frac{f(0)}{g(0)} \geq 1$, then $X_{(r, n, m, k)} \leq{ }_{D C R Q E} Y_{(r, n, m, k)}$.

Proof. According to the definition of convex order, we have

$$
\frac{f(x)}{g\left[G^{-1}(F(x))\right]} \geq \frac{f(0)}{g(0)} \geq 1 .
$$

Since $G_{Y_{(r, n, m, k)}}(x)=\psi_{r: n}(G(x))$, then $G_{Y_{(r, n, m, k)}}^{-1}(x)=G^{-1}\left(\psi_{r: n}^{-1}(x)\right)$. Therefore

$$
\begin{aligned}
G_{Y_{(r, n, m, k)}}^{-1}\left(F_{X_{(r, n, m, k)}}(x)\right) & =G^{-1}\left[\psi_{r: n}^{-1}\left(F_{X_{(r, n, m, k)}}(x)\right)\right] \\
& =G^{-1}\left[\psi_{r: n}^{-1}\left(\psi_{r: n}(F(x))\right)\right] \\
& =G^{-1}[F(x)] .
\end{aligned}
$$

But

$$
\begin{aligned}
g_{Y_{(r, n, m, k)}}\left[G_{Y_{(r, n, m, k)}}^{-1}\left(F_{X_{(r, n, m, k)}}(x)\right)\right] & =g_{Y_{(r, n, m, k)}}\left[G^{-1}(F(x))\right] \\
& =\phi_{r: n}(F(x)) g\left[G^{-1}(F(x))\right] .
\end{aligned}
$$

Thus

$$
\frac{f_{X_{(r, n, m, k)}}(x)}{g_{Y_{(r, n, m, k)}}\left[G_{Y_{(r, n, m, k)}}^{-1}\left(F_{X_{(r, n, m, k)}}(x)\right)\right]}=\frac{f(x)}{g\left[G^{-1}(F(x))\right]} \geq 1
$$

where the last inequality follows from (3.7). We must now prove that

$$
\int_{t}^{\infty} \bar{F}_{X_{(r, n, m, k)}}(x) \ln \left[\frac{\bar{F}_{X_{(r, n, m, k)}}(t)}{\bar{F}_{X_{(r, n, m, k)}}(x)}\right]\left[\frac{f_{X_{(r, n, m, k)}}(x)}{g_{Y_{(r, n, m, k)}}\left[G_{Y_{(r, n, m, k)}}^{-1}\left(F_{X_{(r, n, m, k)}}(x)\right)\right]}-1\right] d x \geq 0,
$$


From (3.8), we have

$$
\left[\frac{f_{X_{(r, n, m, k)}}(x)}{g_{Y_{(r, n, m, k)}}\left[G_{Y_{(r, n, m, k)}}^{-1}\left(F_{X_{(r, n, m, k)}}(x)\right)\right]}-1\right] \geq 0 .
$$

Since the survival function is nonnegative and decreasing, we see that $\bar{F}_{X_{(r, n, m, k)}}(x) \ln \left[\frac{\bar{F}_{X_{(r, n, m, k)}(t)}}{\bar{F}_{X_{(r, n, m, k)}}(x)}\right] \geq 0$ for each $x>t$. Now we see that the inequality (3.9) is valid, therefore the proof is complete.

\section{Stochastic comparisons between spacings of generalized order statistics}

Here, we state conditions on distributions and parameters required for comparisons between the simple (normalized) spacings of generalized order statistics based on a common distribution as well as based on two distributions.

In view of Theorem 3.1 of [11] and diagram (2.3), we have the following corollary.

Corollary 4.1. Let $\left\{X_{\left(r, n, \widetilde{m}_{n}, k\right)}, r=1, \cdots, n\right\}$ and $\left\{Y_{\left(r, n, \widetilde{m}_{n}, k\right)}, r=1, \cdots, n\right\}$ be the generalized order statistics based on strictly increasing continuous distribution functions $F$ and $G$, respectively. If $m_{i} \geq-1$, for each $i$ and $X_{\left(1, n, \widetilde{m}_{n}, k\right)} \leq_{D C R Q E} Y_{\left(1, n, \widetilde{m}_{n}, k\right)}$, then

$$
U_{i, n} \leq_{i c x} V_{i, n}, \text { for } i=1, \cdots, n .
$$

In the following, we prove a useful lemma.

Lemma 4.1. Let $\left\{X_{\left(r, n, \widetilde{m}_{n}, k\right)}, r=1, \cdots, n\right\}$ be generalized order statistics based on an absolutely continuous distribution function $F$. Further, let $\mu_{1, n}(t)$ denote the mean residual life function of $X_{\left(1, n, \widetilde{m}_{n}, k\right)}$. If $F$ is DMRL $(I M R L)$, then $\mu_{1, n}(t) \geq(\leq) \frac{\mu(t)}{\gamma_{1, n}}, \forall t$, where $\mu(t)$ denotes the mean residual life function of $X$.

Proof. We know that the survival function of $X_{\left(1, n, \widetilde{m}_{n}, k\right)}$ is given by

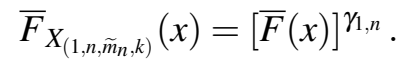

Since $\gamma_{1, n} \geq 1$, the desired result is obtained by a proof similar to the proof of Lemma 2.5 in [19].

The following theorem provides sufficient conditions for ordering the variance of simple spacings of generalized order statistics. This theorem generalizes the results given in [26] in Theorem 3 $(a)$ and $(b)$ [taking into account [27]].

Theorem 4.1. Let $X_{\left(1, n, \widetilde{m}_{n}, k\right)}, \cdots, X_{\left(n, n, \widetilde{m}_{n}, k\right)}$ be generalized order statistics based on an absolutely continuous distribution function $F$, where $m_{i} \geq-1$ for each i. If $X_{\left(1, n, \widetilde{m}_{n}, k\right)}$ is IMRL and $E\left(X^{2}\right)<\infty$, then

(i) $\operatorname{var}\left(U_{r+1, n}\right) \geq \operatorname{var}\left(U_{r, n}\right)$, if $m_{1} \geq \cdots \geq m_{n}$.

(ii) $\operatorname{cov}\left(U_{r, n}, U_{r+1, n}\right) \geq 0$. 
Proof. (i) follows immediately from Lemma 2.1 (ii), Theorem 3.1 and Lemma 2 in [40] and [26], respectively.

To prove $(i i)$, let $\mu_{1, n-i}($.$) denote the mean residual life function of X_{\left(1, n-i, \widetilde{m}_{i, n}^{*}, k\right)}$. Then

$$
\begin{aligned}
E & \left(X_{\left(i+1, n, \widetilde{m}_{n}, k\right)}-X_{\left(i, n, \widetilde{m}_{n}, k\right)} \mid X_{\left(i, n, \widetilde{m}_{n}, k\right)}=t\right) \\
& =\int_{0}^{\infty} P\left(X_{\left(i+1, n, \widetilde{m}_{n}, k\right)}-X_{\left(i, n, \widetilde{m}_{n}, k\right)}>x \mid X_{\left(i, n, \widetilde{m}_{n}, k\right)}=t\right) d x \\
& =\int_{0}^{\infty} P\left(X_{\left(i+1, n, \widetilde{m}_{n}, k\right)}>x+t \mid X_{\left(i, n, \widetilde{m}_{n}, k\right)}=t\right) d x \\
& =\int_{0}^{\infty}\left(\frac{\bar{F}(x+t)}{\bar{F}(t)}\right)^{\gamma_{i+1, n}} d x \\
& =\frac{\int_{t}^{\infty}(\bar{F}(x))^{\gamma_{i+1, n}} d x}{(\bar{F}(t))^{\gamma_{i+1, n}}} \\
& =\mu_{1, n-i}(t),
\end{aligned}
$$

where the third and fourth equalities follow from (2.1) and substituting $x$ for $x+t$, respectively. Since the survival function of $X_{\left(1, n-i, \widetilde{m}_{i, n}^{*}, k\right)}$ is given by

$$
\bar{F}_{X_{\left(1, n-i, \tilde{m}_{i, n}^{*}, k\right)}}(x)=(\bar{F}(x))^{\gamma_{i+1, n}},
$$

the last equality follows from (4.2) and the definition of a mean residual life function. The rest of the proof is similar to that of Theorem 3 in [26] and hence omitted. This completes the proof.

The following theorem generalized Theorem $4(a)$ in [26] (see also [27]) that concerns variances of successive generalized order statistics.

Theorem 4.2. Let $X_{\left(1, n, \widetilde{m}_{n}, k\right)}, \cdots, X_{\left(n, n, \widetilde{m}_{n}, k\right)}$ be generalized order statistics based on absolutely continuous distribution function $F$, where $m_{i} \geq-1$ for each $i$. Further, suppose that $X_{\left(1, n, \widetilde{m}_{n}, k\right)}$ is IMRL and $E\left(X^{2}\right)<\infty$, then

$$
\operatorname{var}\left(X_{\left(i+1, n, \widetilde{m}_{n}, k\right)}\right) \geq \operatorname{var}\left(X_{\left(i, n, \widetilde{m}_{n}, k\right)}\right) .
$$

Proof. Suppose that $X_{\left(1, n, \widetilde{m}_{n}, k\right)}$ is IMRL. As in the proof of Theorem 4 part (a) of [26], we have

$$
\begin{aligned}
& \operatorname{var}\left(X_{\left(i+1, n, \widetilde{m}_{n}, k\right)}\right)=\operatorname{var}\left(X_{\left(i+1, n, \widetilde{m}_{n}, k\right)}-X_{\left(i, n, \widetilde{m}_{n}, k\right)}\right) \\
& \quad+\operatorname{var}\left(X_{\left(i, n, \widetilde{m}_{n}, k\right)}\right)+2 \operatorname{cov}\left(X_{\left(i+1, n, \widetilde{m}_{n}, k\right)}-X_{\left(i, n, \widetilde{m}_{n}, k\right)}, X_{\left(i, n, \widetilde{m}_{n}, k\right)}\right),
\end{aligned}
$$

but

$$
\begin{aligned}
& \operatorname{cov}\left(X_{\left(i, n, \widetilde{m}_{n}, k\right)}, X_{\left(i+1, n, \widetilde{m}_{n}, k\right)}-X_{\left(i, n, \widetilde{m}_{n}, k\right)}\right) \\
& \quad=\operatorname{cov}\left(X_{\left(i, n, \widetilde{m}_{n}, k\right)}, E\left(X_{\left(i+1, n, \widetilde{m}_{n}, k\right)}-X_{\left(i, n, \widetilde{m}_{n}, k\right)} \mid X_{\left(i, n, \widetilde{m}_{n}, k\right)}\right)\right) \\
& \quad=\operatorname{cov}\left(X_{\left(i, n, \widetilde{m}_{n}, k\right)}, \mu_{1, n-i}\left(X_{\left(i, n, \widetilde{m}_{n}, k\right)}\right)\right) \\
& \quad \geq 0
\end{aligned}
$$

Since $X_{\left(1, n, \widetilde{m}_{n}, k\right)}$ is IMRL, by Lemma 2.1(i), we conclude that $X_{\left(1, n-i, \widetilde{m}_{i, n}^{*}, k\right)}$ is also IMRL. Therefore $\mu_{1, n-i}(t)$ is increasing in $t$. Since the covariance between two increasing functions of a random variable is nonnegative, the inequality (4.3) is obtained. So, the theorem is proved. 
Theorem 4.3. Let $X_{\left(1, n, \widetilde{m}_{n}, k\right)}, \cdots, X_{\left(n, n, \widetilde{m}_{n}, k\right)}$ be generalized order statistics based on absolutely continuous distribution function $F$. If $F$ is IMRL $(D M R L)$ and $E\left(X^{2}\right)<\infty$, then

$$
\operatorname{var}\left(X_{\left(1, n, \widetilde{m}_{n}, k\right)}\right) \leq(\geq) \frac{1}{\gamma_{1, n}^{2}} \operatorname{var}(X) .
$$

Proof. It is similar to Theorem 4 (b) of [26] [taking into account [27]] and hence is omitted.

In replacement and repair strategies, although the shape of the hazard rate function plays an important role, the mean residual life function is found to be more relevant than the hazard rate function because the former summarizes the entire residual life function, whereas the latter involves only the risk of instantaneous failure at some time t. Therefore, in some situations, it may be more appropriate to use the mean residual life function to compare the lifetime of two devices. For a comprehensive review on mean residual life function and its various applications in parametric as well as nonparametric contexts the reader is referred to [29] and references therein. The following theorem establishes the mean residual life order among normalized spacings of two samples of generalized order statistics, when the distribution functions are ordered by mean residual life order and one of them has an increasing mean residual life function while the other has a decreasing mean residual life function. This theorem generalizes Theorem 3.3 in [19].

Theorem 4.4. Let $X \leq_{m r l} Y$ and $X$ and $Y$ are IMRL and DMRL, respectively; Then

$$
\widetilde{U}_{i, n} \leq_{m r l} \widetilde{V}_{j, m} \text {, for } i=1, \cdots, n, j=1, \cdots, m .
$$

Proof. For each $i$, let $\eta_{i, n}^{X}(t)$ and $\zeta_{i, n}^{X}(t)$ denote the mean residual life function of $U_{i, n}$ and $\widetilde{U}_{i, n}$ at $t \in \mathbb{R}^{+}$, respectively. Also let $\mu_{1, n-i}^{X}(t)$ denote the mean residual life function of $X_{\left(1, n-i, \widetilde{m}_{i, n}^{*}, k\right)}$ at $t \in \mathbb{R}^{+}$, where $\widetilde{m}_{i, n}^{*}=\left(m_{i+1}, \cdots, m_{n-1}\right)$. The corresponding notations for random variable $Y$ are $\eta_{j, m}^{Y}(t), \zeta_{j, m}^{Y}(t)$ and $\mu_{1, m-j}^{Y}(t)$. As shown in [40],

$$
\begin{gathered}
\eta_{i, n}^{X}(t)=E\left[\mu_{1, n-i+1}^{X}\left(t+U_{1}\right)\right], t \in \mathbb{R}^{+}, \\
\eta_{j, m}^{Y}(t)=E\left[\mu_{1, m-j+1}^{Y}\left(t+U_{2}\right)\right], t \in \mathbb{R}^{+},
\end{gathered}
$$

where $U_{1}$ and $U_{2}$ are random variables. Then

$$
\zeta_{i, n}^{X}(t)=\gamma_{i, n} \eta_{i, n}^{X}\left(\frac{t}{\gamma_{i, n}}\right)
$$

and

$$
\zeta_{j, m}^{Y}(t)=\gamma_{j, m} \eta_{j, m}^{Y}\left(\frac{t}{\gamma_{j, m}}\right)
$$

The rest of the proof is similar to that of Theorem 3.3 in [19] and hence omitted.

The following corollary follows from Theorem 4.4 and Proposition 2.1.

Corollary 4.2. Under the same conditions as in Theorem 4.4, if, in addition, $X_{\left(1, n, \widetilde{m}_{n}, k\right)}$ or $Y_{\left(1, m, \widetilde{m}_{m}, k\right)}$ or both are IMRL, then

$$
\widetilde{U}_{i, n} \leq_{e w} \widetilde{V}_{j, m}, \quad \text { for } i=1, \cdots, n, j=1, \cdots, m .
$$

Remark 4.1. If $X$ is DFR(IFR), then $X_{\left(1, n, \widetilde{m}_{n}, k\right)}$ is IMRL(DMRL). 
In many practical applications such as biological science, physics, engineering and manufacture, the available data can be interpreted as lifetimes and it is important to predict future observations. So probability distributions are often used in survival analysis for modeling data, because they offer insight into the nature of various parameters and functions.

The following examples illustrate some situations where Theorems 4.1-4.4 and Corollary 4.2 are applicable.

- The generalized modified Weibull distribution $(\operatorname{GMW}(\alpha, \gamma, \lambda, \beta))$ is introduced in [12] that contains, as special sub-models, the Weibull, exponentiated exponential, exponentiated Weibull, generalized Rayleigh, modified Weibull and some other distributions. For $\gamma \geq 1$ and $0<\beta<1(0<\gamma<1$ and $\beta>1)$, this distribution is IFR (DFR).

- A new DFR lifetime distribution is introduced in [13] by combining a power series distribution truncated at zero with some exponential distributions and is named the exponentialpower series (EPS) distribution. This distribution includes as special cases, lifetime distributions presented in [1], [30] and [38]. The hazard rate function is decreasing for all elements of the power series distributions.

- A new lifetime class with decreasing failure rate, called the Poisson lifetime distribution (PL), is introduced in [2]. It is obtained by compounding truncated Poisson distribution and a lifetime distribution $f_{Y}(y)$.

- The exponential-Weibull distribution is proposed in [14] which is denoted by $\operatorname{EW}(\alpha, \beta, \gamma)$ and for $\gamma<1(\gamma>1)$ is DFR (IFR).

- The exponentiated exponential-Poisson distribution (EEP $(\beta, \lambda, \alpha))$ is introduced in [35]. The hazard rate function is decreasing (increasing) for $0<\alpha \leq 1(\alpha>1$ and $0<\lambda<$ $1-1 / \alpha)$.

- A general family of continuous lifetime distributions is obtained by compounding any continuous distribution function $G($.$) and the Poisson-Lindley distribution. This family$ is named PoissonLindley-G distribution and introduced in [3]. If the hazard rate function of $G($.$) , i.e. h_{g}(x)=g(x) /[\bar{G}(x)]$ is decreasing, then the hazard rate function of it is also decreasing. The Weibull PoissonLindley (WPL) distribution, the Burr PoissonLindley (BPL) distribution, the exponentiated Weibull PoissonLindley (EWPL) distribution and the Dagum PoissonLindley (DPL) distribution are some special cases of this distribution.

- The generalized linear failure rate distribution $(\operatorname{GLFRD}(a, b, \theta))$ is introduced in [36]. The Linear failure distribution, Generalized exponential distribution and Generalized Rayleigh distribution are some special cases of this distribution. One can easily verify that: (i) if $\theta=1$, the hazard function is either increasing (if $b>0$ ) or constant (if $b=0$ and $a>0$ ); (ii) when $\theta>1$, the hazard function is always increasing; and (iii) if $\theta<1$, then the hazard function will be either decreasing if $b=0$ or bathtub if $b>0$.

\section{Concluding remarks}

Generalized order statistics have been of interest in reliability theory, statistical modelling and inference because of their flexibility. They unify the study of several cases of ordered random variables. In this paper, we have obtained several results concerning the comparison of generalized order statistics in terms of the dynamic cumulative residual quantile entropy order and proved that when $X_{\left(1, n, \widetilde{m}_{n}, k\right)}$ is IMRL, the spacings of generalized order statistics are ordered by variance, and that the 
covariance between successive spacings is nonnegative. We also compared the variance of successive generalized order statistics. Finally, we compared the normalized spacings of generalized order statistics based on different distribution functions in terms of the mean residual life order and gave examples to illustrate situations where the theorems are applicable.

\section{Acknowledgments}

The authors grateful to the editorial board of Journal of Statistical Theory and Applications (JSTA), in particular, Professor Hamedani and would like to thank two anonymous referees for useful comments and suggestions that lead to this improved version of the paper.

\section{References}

[1] K. Adamidis and S. Loukas, A lifetime distribution with decreasing failure rate, Statist. Probab. Lett. 39 (1998) 35-42.

[2] S. Alkarni and A. Oraby, A compound class of Poisson and lifetime distributions, J. Stat. Appl. Probab. 1 (2012) 45-51.

[3] A. Asgharzadeh, H. S. Bakouch, S. Nadarajah and L. Esmaeili, A new family of compound lifetime distributions, Kybernetika 50 (2014) 142-169.

[4] N. Balakrishnan, E. Cramer and U. Kamps, Bounds for means and variances of progressive Type II censored order statistics, Statist. Probab. Lett. 54 (2001) 301-315.

[5] N. Balakrishnan and C.R. Rao (eds.), Handbook of statistics, (Vol. 16: Theory and Methods. Amsterdam: North-Holland, 1998a).

[6] N. Balakrishnan and C.R. Rao, (eds.), Handbook of statistics, (Vol. 17: Applications. Amsterdam: North-Holland, 1998b).

[7] N. Balakrishnan and P. Zhao, Ordering properties of order statistics from heterogeneous populations: a review with an emphasis on some recent developments, Probab. Engrg. Inform. Sci. 27 (2013) 403-443.

[8] R.E. Barlow and F. Proschan, Statistical Theory of Reliability and Life Testing, (Probability Models. To Begin With, Silver Spring, MD, 1981).

[9] F. Belzunce, J.A. Mercader and J.M. Ruiz, Stochastic comparisons of generalized order statistics, Probab. Engrg. Inform. Sci. 19 (2005) 99-120.

[10] F. Belzunce and C. Martnez-Riquelme, Some results for the comparison of generalized order statistics in the total time on test and excess wealth orders, Statist. Papers 56 (2015) 1175-1190.

[11] F. Belzunce, C. Martnez-Riquelme, J. M. Ruiz and M.A. Sordo, On sufficient conditions for the comparison in the excess wealth order and spacings, J. Appl. Probab. 53 (2016) 33-46.

[12] J.M.F. Carrasco, E.M.M. Ortega and G.M. Cordeiro, A generalized modified Weibull distribution for lifetime modeling, Comput. Stat. Data. Anal. 53 (2008) 450-462.

[13] M. Chahkandi and M. Ganjali, on some lifetime distributions with decreasing failure rate, Comput. Stat. Data. Anal. 53 (2009) 4433-4440.

[14] G.M. Cordeiro, E.M. Ortega and A.J. Lemonte, The exponential-Weibull lifetime distribution, J. Stat. Comput. Simul. 84 (2014) 2592-2606.

[15] E. Cramer, U. Kamps and T. Rychlik, On the existence of moments of generalized order statistics, Statist. Probab. Lett. 59 (2002) 397-404.

[16] Z. Fang, T. Hu, Y. Wu and W. Zhuang, Multivariate stochastic orderings of spacings of generalized order statistics, Chinese J. Appl. Probab. Statist. 22 (2006) 295-303.

[17] M. Franco, J.M. Ruiz and M.C. Ruiz, Stochastic orderings between spacings of generalized order statistics, Probab. Engrg. Inform. Sci. 16 (2002) 471-484.

[18] L. Gajek and A. Okolewski, Sharp bounds on moments of generalized order statistics, Metrika 52 (2000) 27-43. 
[19] T. Hu and Y. Wei, Stochastic comparisons of spacings from restricted families of distributions, Statist. Probab. Lett. 53 (2001) 91-99.

[20] T. Hu and W. Zhuang, Stochastic properties of $p$-spacings of generalized order statistics, Probab. Engrg. Inform. Sci. 19 (2005) 257-276.

[21] U. Kamps, a Concept of Generalized Order Statistics, (Teubner, Stuttgart, 1995a).

[22] U. Kamps, A concept of generalized order statistics, Journal of Statistical Planning and Inference 48 (1995b) 1-23.

[23] D.T. Kang and L. Yan, On the dynamic cumulative residual quantile entropy ordering, Stat. Methodol. 32 (2016) 14-35.

[24] C. Keseling, Conditional distributions of generalized order statistics and some characterizations, Metrika 49 (1999) 27-40.

[25] B.E. Khaledi, Some new results on stochastic comparisons between generalized order statistics, JIRSS 4 (2005) 35-49.

[26] S.N.U.A. Kirmani, On sample spacings from IMRL distributions, Statist. Probab. Lett. 29 (1996) 159166.

[27] S.N.U.A. Kirmani, Erratum On sample spacings from IMRL distributions, Statist. Probab. Lett. 37 (1998) 315.

[28] S. Kochar, Stochastic comparisons of order statistics and spacings: a review, ISRN Probability and Statistics 2012 (2012).

[29] P.R. Krishnan and C.R. Rao, Handbook of statistics, Elsevier, Amsterdam 7 (1988) 215-224.

[30] C. Kus, A new lifetime distribution, Comput. Stat. Data. Anal. 51 (2007) 4497-4509.

[31] X. Li, A note on expected rent in auction theory, Oper. Res. Lett. 33 (2005) 531-534.

[32] D. Nasri-Roudsari, Extreme value theory of generalized order statistics, J. Statist. Plann. Inference 50 (1996) 281-297.

[33] A. Paul and G. Gutierrez, Mean sample spacings, sample size and variability in an auction-theoretic framework, Oper. Res. Lett. 32 (2004) 103-108.

[34] G. Qiu and J. Wang, Some comparisons between generalized order statistics, Appl. Math 22 (2007) 325-333.

[35] M.M. Risti'c and S. Nadarajah, A new lifetime distribution, J. Stat. Comput. Simul. 84 (2014) 135-150.

[36] A.M. Sarhan and D. Kundu, Generalized linear failure rate distribution, Comput. Stat. Data. Anal. 55 (2011) 644-654.

[37] M. Shaked and J.G. Shanthikumar, Stochastic orders, (New York: Springer, 2007).

[38] R. Tahmasbi and S. Rezaei, A two-parameter lifetime distribution with decreasing failure rate, Comput. Stat. Data. Anal. 52 (2008) 3889-3901.

[39] H. Xie and T. Hu, Ordering p-spacings of generalized order statistics revisited, Probab. Engrg. Inform. Sci. 23 (2009) 1-16.

[40] H. Xie and W. Zhuang, Some new results on ordering of simple spacings of generalized order statistics, Probab. Engrg. Inform. Sci. 25 ( 2011 ) 71-81. 